\title{
Spatial-Temporal Instability of an Inviscid Shear Layer
}

\author{
Qing-fei Fu, Li-zi Qin, and Li-jun Yang \\ School of Astronautics, Beihang University, Beijing 100191, China \\ Correspondence should be addressed to Li-jun Yang; 13426315077@163.com \\ Received 23 November 2016; Revised 11 March 2017; Accepted 22 March 2017; Published 9 April 2017 \\ Academic Editor: Corin Segal
}

Copyright (C) 2017 Qing-fei Fu et al. This is an open access article distributed under the Creative Commons Attribution License, which permits unrestricted use, distribution, and reproduction in any medium, provided the original work is properly cited.

\begin{abstract}
In this work, we explore the transition of absolute instability and convective instability in a compressible inviscid shear layer, through a linear spatial-temporal instability analysis. From linearized governing equations of the shear layer and the ideal-gas equation of state, the dispersion relation for the pressure perturbation was obtained. The eigenvalue problem for the evolution of twodimensional perturbation was solved by means of shooting method. The zero group velocity is obtained by a saddle point method. The absolute/convective instability characteristics of the flow are determined by the temporal growth rate at the saddle point. The absolute/convective nature of the flow instability has strong dependence on the values of the temperature ratio, the velocity ratio, the oblique angle, and $M$ number. A parametric study indicates that, for a great value of velocity ratio, the inviscid shear layer can transit to absolute instability. The increase of temperature ratio decreases the absolute growth rate when the temperature ratio is large; the effect of temperature ratio is opposite when the temperature ratio is relatively small. The obliquity of the perturbations would cause the increase of the absolute growth rate. The effect of $M$ number is different when the oblique angle is great and small. Besides, the absolute instability boundary is found in the velocity ratio, temperature ratio, and $M$ number space.
\end{abstract}

\section{Introduction}

A high mixing rate of the fuel and air is desired in scramjet engine for the propulsion of hypersonic aircraft, because the residence time of the fuel and air in the combustion chamber is very short. In the interest of the projected use of the scramjet engine, it is fundamental and also extremely important to understand the stability characteristics of compressible shear/mixing layers. Many experimental studies [1-3] suggested that the mixing rates of shear layers decrease as the Mach number increases from zero. Hence, a major concern in the development of scramjet is the mixing enhancement techniques. Imparting disturbances on the shear layer, which pulsate at some prescribed frequency, is a choice of mixing enhancement techniques. The prescribed frequency can be obtained through linear stability analysis of the compressible shear layer.

There have been numerous literatures on the topic of linear stability analysis of the compressible shear layer, including the earlier studies conducted by Lessen et al. $[4,5]$ Drazin and Davey [6] performed a temporal stability analysis of a compressible mixing layer, which has a hyperbolic tangent velocity profile and uniform temperature throughout the layer. Jackson and Grosch [7] reported the results of the inviscid spatial stability of a parallel compressible mixing layer. All these studies found multiple stability modes. Zhuang et al. [8] and Ragab [9] both found a strong stabilization effect on the flow when increasing Mach number. Ho and Huerre [10] summarized the studies on linear stability analysis of incompressible shear layer. For the studies on linear stability analysis of compressible shear layer, the reader can refer to the relevant literature in the monograph by Criminale et al. [11].

The studies above are all confined in the scope of temporal or spatial mode. When we study the stability characteristics of shear layers in spatial-temporal mode, which treat both spatial and temporal eigenvalue complex [12, 13], there are two distinct instabilities for spatial-temporal evolving disturbances: convective and absolute instabilities. The concept of absolute and convective instabilities was first put forward by Briggs [14] in the study of plasma instability and then introduced to classical hydrodynamic stability [15]. A flow is convectively unstable if the unsteady response to an impulsive perturbation grows along some rays that pass away from the 


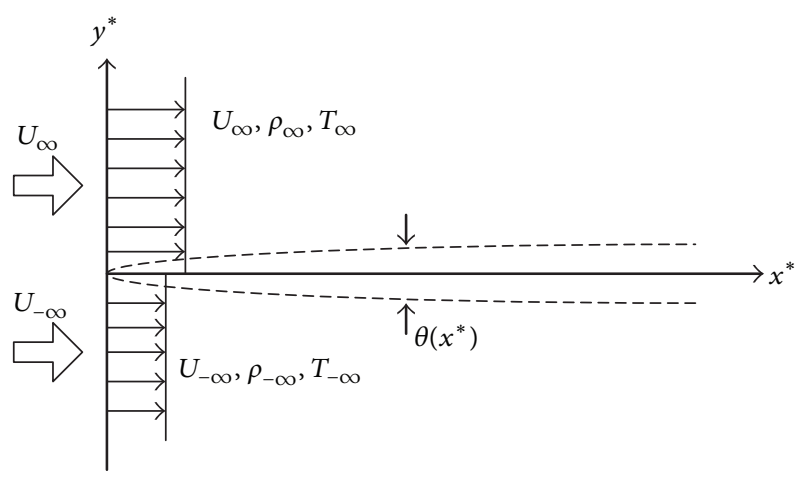

Figure 1: Schematic of a shear layer between two streams.

forcing location but decays at the forcing location itself. A flow is absolutely unstable if the impulse response grows at the forcing location [16].

An absolutely unstable flow is not sensitive to external disturbances and initial conditions; consequently, the "flow management" techniques such as forcing the shear layer at some prescribed frequency could be useless. [17]. Thus, if we want to control the downstream evolution of the flow, it is essential to determine whether the shear/mixing layer is convectively or absolutely unstable. Kulikovskii and Shikina [18-20] studied the asymptotic behavior of localized perturbations on the surface of a shear discontinuity separating two homogeneous steady flows of ideal incompressible fluid in the linear approximation. The effect of surface tension, gravity forces, and viscosity is taken into account. Pavithran and Redekopp [21] investigated the absolute and convective instabilities for a subsonic mixing layer with the hyperbolictangent-like profiles in velocity and temperature fields. The study on transition of absolute and convective instability for compressible shear layer is rare. Caillol [22] analyzed the transition of absolute and convective instabilities for an inviscid mixing layer. The Mach number in his work attained high supersonic values. However, he did not consider the density stratification within the mixing layer. Large density stratification is possible for binary mixing layers at high pressures, which is usually encountered in power and propulsion system.

The aim of this paper is to track the transition between the absolute instability and convective instability in a compressible shear layer. The density stratification is taken into account. The effects of flow parameters on the spatialtemporal stability of a compressible shear layer are examined by observing whether an increase of the value of parameter tends to increase or decrease the value of absolute growth rate.

\section{Theoretical Framework}

The base flow is a shear layer between two streams with its far-stream condition denoted by the subscripts $\infty$ and $-\infty$, as shown in Figure 1. The thickness of shear layer increases with increasing distance downstream, denoting that the base flow is nonparallel. Here, we made a locally parallel flow assumption, implying that the present results yield the instability characteristics of individual profiles to leading order when the shear layer thickness grows slowly with downstream distance [23]. The streamwise direction is defined as $x$ and the cross stream is defined as $y$. In the present study, $U$ denotes the velocity, $\rho$ the density, and $T$ the temperature. Taking the momentum thickness $\theta$ and average mean velocity $\bar{U}=\left(U_{\infty}+U_{-\infty}\right) / 2$ as the characteristic length and velocity scale, respectively, the flow properties can be normalized. Superscript $*$ denotes a nondimensional quantity.

The linear stability analysis, the features of which are standard and, hence, are not presented in detail here, is performed for a flow governed by the conservation equations of continuity, momentum, and energy. The thermodynamic condition of the layer is assumed far above the critical point, so the ideal-gas equation of state is used to close the formulation. Linearization of these equations starts by splitting the dependent variables into base and perturbation components. A base flow is assumed that is described by the normalized velocity profile $\bar{u}^{*}\left(y^{*}\right)$ and temperature profile $\bar{T}^{*}\left(y^{*}\right)$ specified as (1)-(2) and by a constant thermodynamic pressure [24]:

$$
\begin{aligned}
& \bar{u}^{*}=1+\Lambda \tanh \left(\frac{y^{*}}{2}\right) \\
& \bar{T}^{*}=\frac{S_{T}+1}{2}+\frac{S_{T}-1}{2} \tanh \left(\frac{y^{*}}{2}\right),
\end{aligned}
$$

where the velocity ratio is defined as $\Lambda=\left(U_{\infty}-U_{-\infty}\right) /\left(U_{\infty}+\right.$ $U_{-\infty}$ ) and the temperature ratio is defined as $S_{T}=T_{\infty} / T_{-\infty}$. The density distributions are obtained by employing the ideal-gas equation of state on the shear layer with the temperature distribution defined in (2).

We perturbed the steady-state solution of the flow with a small-amplitude wave in normal modes:

$$
\begin{aligned}
& q^{\prime}(x, y, z, t)=\hat{q}(y) \exp [i k(x \cos \phi+z \sin \phi-c t)] \\
& \quad=\widehat{q}(y) \exp [i(k x \cos \phi+k z \sin \phi-\omega t)],
\end{aligned}
$$

where $q^{\prime}$ denotes the perturbation components, $\hat{q}$ denotes the perturbation wave amplitude, $k, c$, and $\omega(\omega=k c)$ are the wave number, phase speed, and frequency, respectively. $\phi$ denotes the angle between the wave propagation direction and the streamwise direction of the mean flow. When the disturbance wave propagates in the axial direction, $\phi$ equals zero, and the resultant system is a two-dimensional problem.

To derive the governing equation for $\widehat{p}^{*}\left(y^{*}\right)$, the governing equations and equation of state are linearized by considering small perturbations to the mean flow whose velocity and temperature profiles are $\bar{u}^{*}\left(y^{*}\right)$ and $\bar{T}^{*}\left(y^{*}\right)$, respectively. By employing a process of elimination, a single 


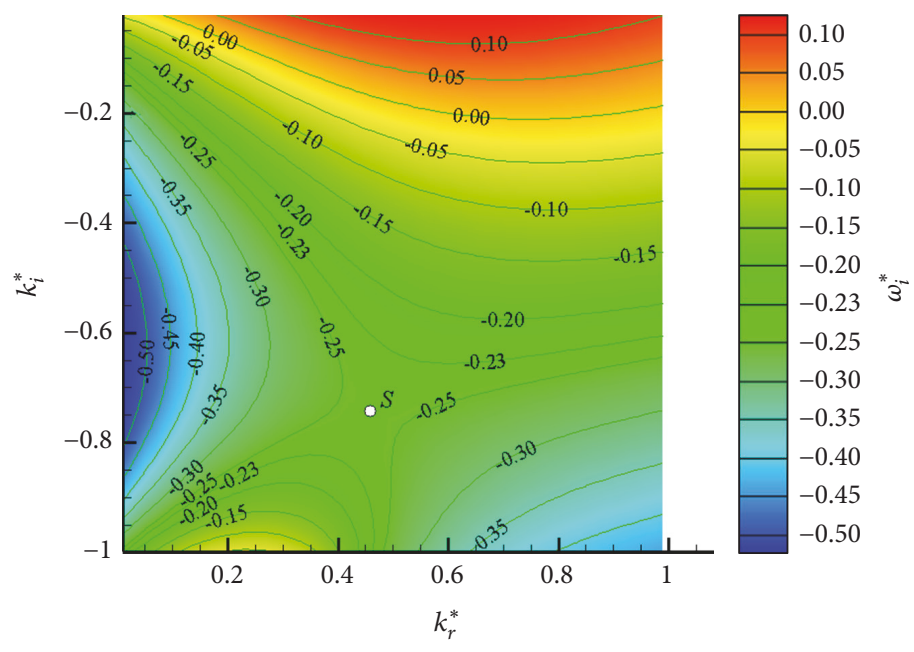

FIgURE 2: Contours of $\omega_{i}^{*}=$ constant in the complex $k^{*}$ plane $\left(\Lambda=1, \phi=0, M=0, S_{T}=5\right)$.

equation can be derived for the radial component of the pressure perturbation $\widehat{p}^{*}[25-28]$ :

$$
\begin{gathered}
\frac{d^{2} \widehat{p}^{*}}{d y^{* 2}}-\left[\frac{2 \bar{u}_{y}^{*} \cos \phi}{\bar{u}^{*} \cos \phi-c^{*}}+\frac{\bar{\rho}_{y}^{*}}{\bar{\rho}^{*}}\right] \frac{d \widehat{p}^{*}}{d y^{*}} \\
-k^{* 2}\left[1-M^{2}\right] \widehat{p}^{*}=0,
\end{gathered}
$$

where $M=(\bar{u} \cos \phi-c) / \bar{a}, \bar{a}$ denotes the speed of sound. For simplicity of notation, the subscript $y$ stands for the spatial derivative with respect to the $y$ coordinate. The governing equation of the two-dimensional incompressible shear layer can be found by setting $\phi=0$ and $M=0$.

Solution of (4) poses an eigenvalue problem due to the presence of the wave parameters, $k^{*}$, and frequency, $\omega^{*}=$ $k^{*} c^{*}$. The boundary conditions are obtained by considering that (i) at far stream the base flow derivatives are zero and that (ii) the solution must be bounded. The solution is obtained by treating the frequency as the eigenvalue for an externally specified wavenumber and by using a shooting iterative procedure. ODE45 in MATLAB is employed to perform the numerical integration of the ordinary differential equation, and the Newton-Raphson shooting method is utilized to perform the iterative shooting to find the eigenvalue. Convergence is found to be strongly dependent on a good starting guess for $\omega^{*}$. The detailed process is as follows: to obtain the $\omega^{*}\left(k^{*}\right)$ relations, we begin from an intermediate $k^{*}$ (e.g., $\left.k^{*}=0.4\right)$ and make an initial guess for $\omega^{*}$. Then, the NewtonRaphson shooting method is used, assuming the initial guess for $\omega^{*}$ is good enough to obtain the exact $\omega^{*}$. Then we decrease or increase $k^{*}$ by $\Delta k^{*}$ and use $\omega^{*}$ calculated with the old $k^{*}$ as the initial guess for the new shooting iteration. If the shooting iteration converges again, we will move to the smaller or larger $k^{*}$. With this computing process, the $\omega^{*}-k^{*}$ curve extends from the middle to both low and high $k^{*}$ regimes. If, at some point, the shooting method diverges or can not obtain a physical solution, or the resultant $\omega^{*}-k^{*}$ curve is not smooth enough, we conclude that the initial guess for $\omega^{*}$ at $k^{*}=0.4$ is incorrect and we provide a new guess and start over again. It is by this process that the $\omega^{*}-k^{*}$ contours presented in this article are obtained.

This study focuses on the transition between absolute instability (AI) and convective instability (CI) for a compressible inviscid shear layer, which are dominated by modes with zero group velocity. These correspond to complex pairs, $\left(k_{0}^{*}, \omega_{0}^{*}\right)$, which satisfy $(4)$ and also the further property that $\partial \omega^{*} / \partial k^{*}=0$.

The saddle point method is used to determine the solution with zero group velocity. In general, there are two distinct spatial branches of solutions of (4) on the complex $k^{*}$ plane. The two branches will approach each other as the growth rate $\omega_{i}^{*}$ is decreased from large positive values, and a saddle point on the complex $k^{*}$ plane will occur at point $k^{*}=k_{0}^{*}$. It is necessary to identify the physical and nonphysical saddle points. The physical saddle point must satisfy the BriggsBers collision criterion [14, 29]. According to the Briggs-Bers collision criterion, the only relevant complex pair $\left(k_{0}^{*}, \omega_{0}^{*}\right)$ is the physical pair, if the two branches come, respectively, from the downstream-propagating branch $k^{*+}\left(\omega^{*}\right)$ and upstreampropagating branch $k^{*-}\left(\omega^{*}\right)$ on the complex $k$ plane. Then if $\omega_{0 i}^{*}<0$ the flow is said to be convectively unstable, while if $\omega_{0 i}^{*}>0$, the flow is said to be absolutely unstable. Normally, $k_{0}^{*}$ is referred to as the absolute wave number and $\omega_{0 i}^{*}$ the absolute growth rate.

\section{Results and Discussion}

In this section, the effects of dimensionless parameters on the spatial-temporal instability of an inviscid shear layer will be checked by taking a parametric study. The complex frequency $\omega^{*}$ is solved as function of the wave number $k^{*}$ and the parameter set $\left(\Lambda, S_{T}, M\right.$, and $\left.\phi\right)$.

The typical contour plots of the imaginary part of the complex frequency $\omega_{i}^{*}$ in the complex wave number $k^{*}$ plane for an inviscid compressible shear layer are shown in Figure 2. Apparently, there is a saddle point in the plot, 


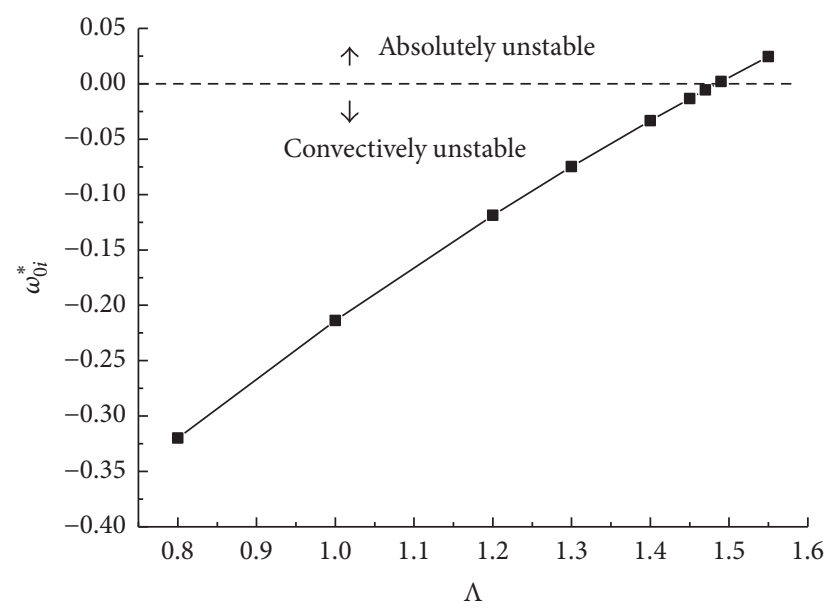

FIGURE 3: The variation of absolute growth rate with velocity ratio $\Lambda$ $\left(\phi=0, M=0, S_{T}=3\right)$.

which is marked with " $S$." The location of the saddle point is $k_{0}^{*}=(0.458,-0.742)$ and the value of real part of complex frequency at the saddle point is $\omega_{0 i}^{*}=-0.245$. The saddle point satisfies the Briggs pinching criterion. In Figure 2, the absolute growth rates are negative, indicating that the inviscid shear layer is convectively unstable with the given dimensionless parameters.

The effect of velocity ratio $\Lambda$ on the absolute instability of an inviscid shear layer is illustrated in Figure 3. It appears in Figure 3 that the absolute growth rate increases with the increases of velocity ratio $\Lambda$. In particular, when the velocity ratio is larger than 1.47 , the absolute growth rate becomes positive. The transition velocity ratio 1.47 was close to the value obtained by Huerre and Monkewitz [12], which was 1.315. Huerre and Monkewitz [12] stated that the mixing layer is absolutely unstable when velocity ratio is larger than 1.315. That is because Huerre and Monkewitz considered shear layer in incompressible fluid, whereas the present paper deals with perfect gas. The velocity ratio represents the shear force imparted on the shear layer; therefore, it can be said that, for a two-dimensional inviscid shear layer, it is absolutely unstable when the shear force imparted on it is relatively large. Manoharan and Hemchandra [23] proposed a mechanism through which absolute instability can cause combustion instability in combustor of power generation and propulsion system. Hence, avoiding the occurrence of absolutely unstable flows in combustors, at least at desired operating conditions, represents a possible passive control strategy to mitigate the occurrence of combustion instability. According to Figure 3, to depress absolute instability, a possible smallest velocity ratio is recommended. However, small velocity ratio, denoting weak shear force imparted on the shear layer, shows no advantage on mixing. Thus the velocity ratio should be designed specially in a combustor.

Figure 4 gives the effects of the temperature ratio, $S_{T}$, on the absolute growth rate of a two-dimensional inviscid shear layer, by increasing $S_{T}$ and keeping other parameters constant for every plot. Figure 4 shows that, for different cases, as the temperature ratio increases, the absolute growth

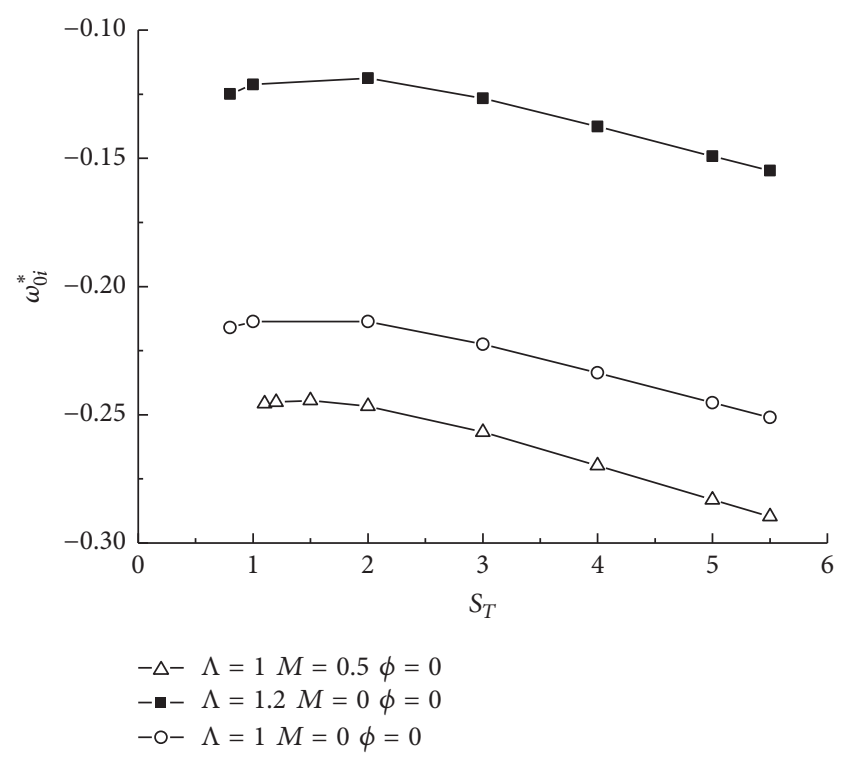

FIGURE 4: The variation of absolute growth rate with temperature ratio $S_{T}$.

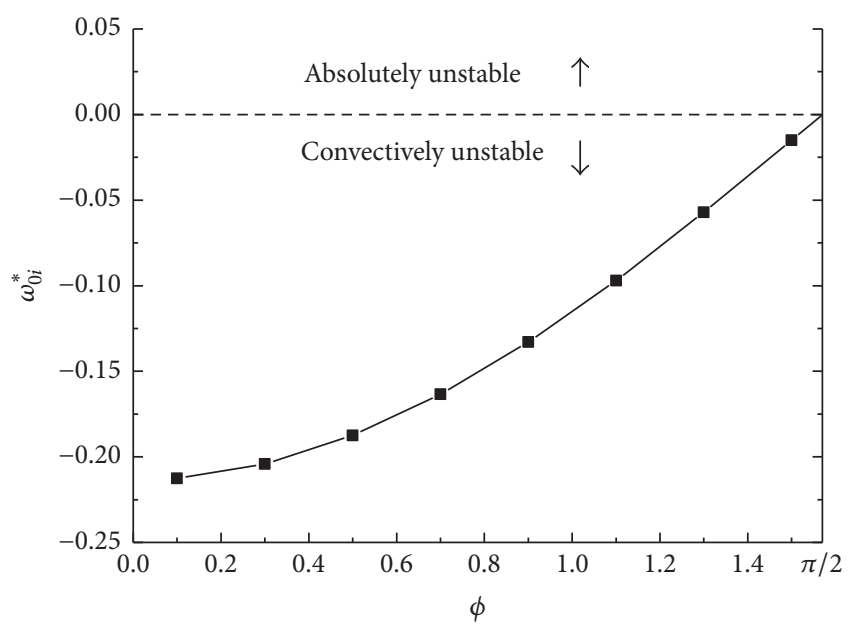

FIGURE 5: The variation of absolute growth rate with oblique angle $\phi\left(\Lambda=1, M=0, S_{T}=2\right)$.

rate decreases. Hence the strategy for suppressing absolute instability is to increase temperature ratio.

However, the temperature effects do not always tend to depress the absolute instability. When the temperature ratio is relatively small $\left(S_{T}<1\right)$, the absolute growth rates shows minor increase with increase of $S_{T}$ for every case. That is to say when $S_{T}$ is relatively large, the effect of $S_{T}$ on absolute growth rate is more remarkable; when $S_{T}$ is small, $S_{T}$ has minor promotion effect on absolute instability of the shear layer.

Now, we check the effect of obliquity of the disturbance wave on the absolute instability by varying the oblique angle, $\phi$. Figure 5 shows the variation of absolute growth rate $\omega_{0 i}^{*}$ with oblique angle $\phi$. It shows in Figure 5 that when the flow is two-dimensional $(\phi=0)$, the absolute growth rate is the smallest. When the oblique angle increases, the absolute 


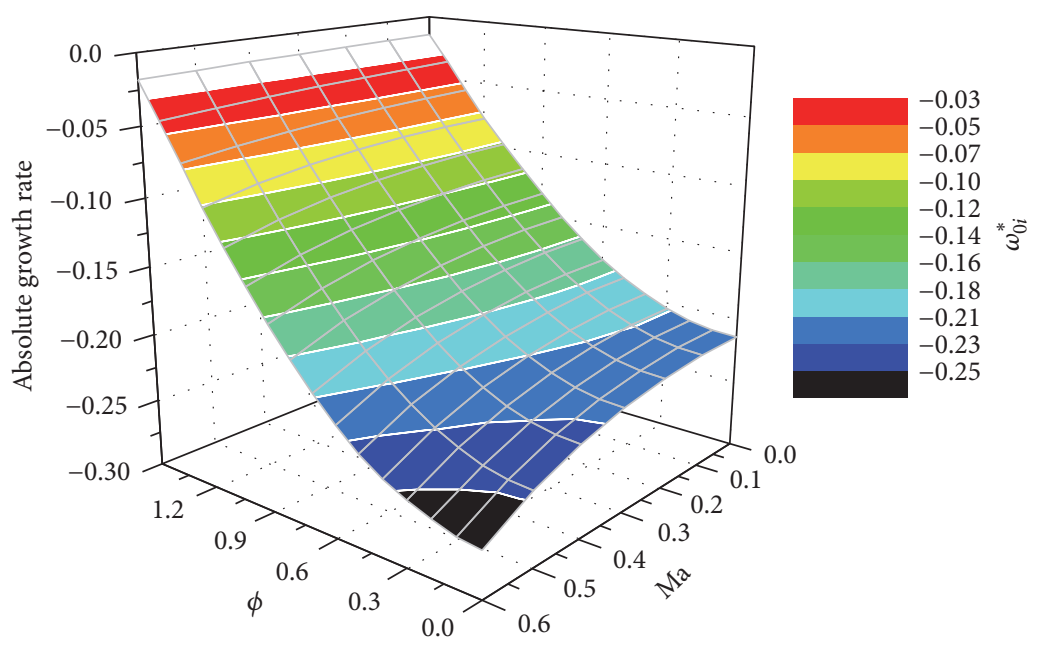

Figure 6: The variation of absolute growth rate with oblique angle and $M$ number $\left(\Lambda=1, S_{T}=2\right)$.

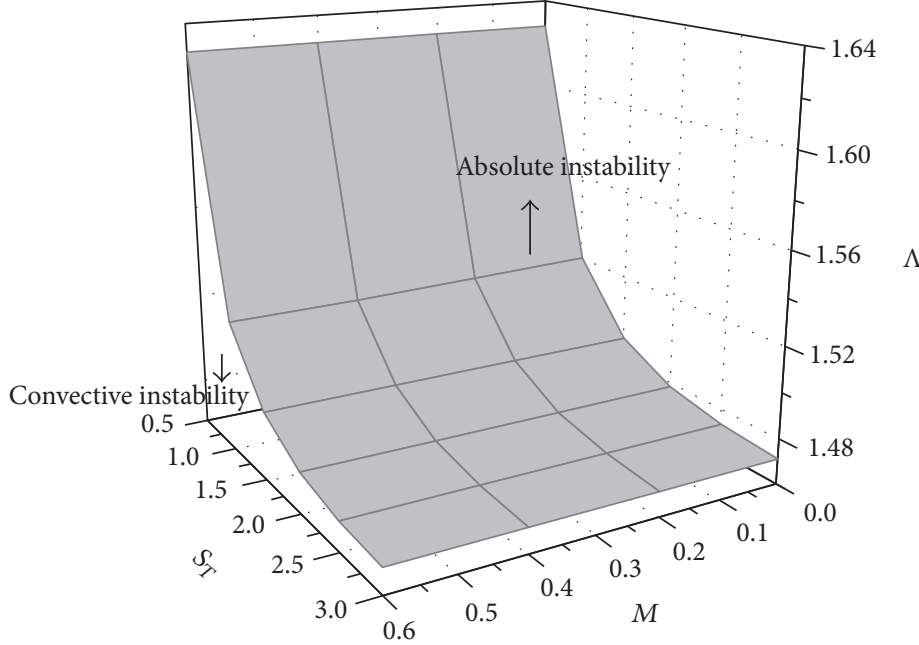

FIgURE 7: The boundary of transition between $\mathrm{AI}$ and $\mathrm{CI}$ in the parameter space of $\Lambda, M$, and $S_{T}(\phi=0)$.

growth rate increases. The increasing rate becomes larger when the oblique angle is greater. However, the absolute growth rate is always negative when $\phi$ varies from 0 to $\pi / 2$. Hence, it can be concluded that the increase of oblique angle can increase the absolute growth rate, and the effect is more remarkable when the oblique angle is greater, or we can say that the three-dimensional disturbance waves are more dominant in inviscid shear layer than two-dimensional wave. But the increasing of $\phi$ would never change the flow into absolute instability.

The parameter $M$ is naturally supposed to be parameter influencing the absolute instability behavior of flow. Here we check the effect of $M$ and oblique angle comprehensively. Figure 6 gives the variation of absolute growth rate with $M$ and oblique angle. It shows that, for a constant $M$, the increase of oblique angle would increase the absolute growth rate, which can also be seen from Figure 5. For a given oblique angle, the effect of $M$ number on absolute growth rate differs when oblique angle is larger or relatively small. When the oblique angle is small, the increase of $M$ would decrease the absolute growth rate; while the oblique angle is greater (close to $\pi / 2$ ), the effect of increasing $M$ is minor. That means the effect of $M$ number is opposite when the disturbances propagate upstream and downstream.

Figure 7 shows the critical value of $\Lambda$ at which the flow transits into absolute instability at different $M$ and $S_{T}$. It can be seen from Figure 7 that the increase of $S_{T}$ would cause the critical value of $\Lambda$ decrease; that is, the increase of $S_{T}$ would expand the absolute instability region in $S_{T}-\Lambda-M$ space. However, the variation of $M$ has no influence on the absolute instability boundary, although the increase of $M$ can decrease the absolute instability growth rate. 


\section{Conclusions}

In this work, we explore the transition of absolute instability and convective instability in a compressible inviscid shear layer, through a linear spatial-temporal instability analysis. From linearized governing equations of the shear layer and the ideal-gas equation of state, the dispersion relation for the pressure perturbation was obtained. The dispersion relation was solved using a shooting iterative procedure. The numerical results show that the increasing velocity ratio can make the inviscid shear layer transition to absolute instability. The increase of temperature ratio decreases the absolute growth rate when the temperature ratio is large; the effect of temperature ratio is opposite when the temperature ratio is relatively small. The obliquity of the perturbations would cause the increase of absolute growth rate. The effect of $M$ number is different when the oblique angle is large or small: when the oblique angle is relatively smaller, the increase of $M$ number would decrease the absolute growth rate; while the oblique angle is relatively large (close to $\pi / 2$ ), the variation of $M$ number has no influence on the absolute growth rate. The absolute instability boundary has been given in the $S_{T}$ $-M-\Lambda$ space. The increase of $S_{T}$ would expand the absolute instability region in the $S_{T}-M-\Lambda$ space, but the variation of $M$ has no influence on the absolute instability boundary.

\section{Conflicts of Interest}

The authors declare that there are no conflicts of interest regarding the publication of this paper.

\section{Acknowledgments}

The financial support of National Nature Science Foundation of China (Support nos. 11672025 and 11302013) is gratefully acknowledged. The authors gratefully acknowledge financial support from China Scholarship Council (Support no. 201506025044).

\section{References}

[1] G. L. Brown and A. Roshko, "On density effects and large structure in turbulent mixing layers," Journal of Fluid Mechanics, vol. 64, no. 4, pp. 775-816, 1974.

[2] N. Chinzei, G. Masuya, T. Komuro, A. Murakami, and K. Kudou, "Spreading of two-stream supersonic turbulent mixing layers," Physics of Fluids, vol. 29, no. 5, pp. 1345-1347, 1986.

[3] D. Papamoschou and A. Roshko, "The compressible turbulent shear layer: an experimental study," Journal of Fluid Mechanics, vol. 197, pp. 453-477, 1988.

[4] M. Lessen, J. A. Fox, and H. M. Zien, "On the inviscid stability of the laminar mixing of two parallel streams of a compressible fluid," Journal of Fluid Mechanics, vol. 23, no. 2, pp. 355-367, 1965.

[5] M. Lessen, J. A. Fox, and H. M. Zien, "Stability of the laminar mixing of two parallel streams with respect to supersonic disturbances," Journal of Fluid Mechanics, vol. 25, no. 4, pp. 737$742,1966$.
[6] P. G. Drazin and A. Davey, "Shear layer instability of an inviscid compressible fluid. Part 3," Journal of Fluid Mechanics, vol. 82, no. 2, pp. 255-260, 1977.

[7] T. L. Jackson and C. E. Grosch, "Inviscid spatial stability of a compressible mixing layer," Journal of Fluid Mechanics, vol. 208, pp. 609-637, 1989.

[8] M. Zhuang, T. Kubota, and P. E. Dimotakis, "On the instability of inviscid, compressible free shear layers," AIAA Paper 19883538, AIAA, Washington, DC, USA, 1988.

[9] S. A. Ragab, "Instabilities in the wake mixing-layer region of a splitter plate separating two supersonic streams," AIAA Paper 1988-3677, AIAA, Washington, DC, USA, 1988.

[10] C.-M. Ho and P. Huerre, "Perturbed free shear layers," Annual Review of Fluid Mechanics, vol. 16, pp. 365-424, 1984.

[11] W. O. Criminale, T. L. Jackson, and R. D. Joslin, Theory and Computation of Hydrodynamic Stability, Cambridge Monographs on Mechanics, Cambridge University Press, Cambridge, UK, 2003.

[12] P. Huerre and P. A. Monkewitz, "Absolute and convective instabilities in free shear layers," Journal of Fluid Mechanics, vol. 159, pp. 151-168, 1985.

[13] L.-J. Yang and Q.-F. Fu, "Stability of confined gas-liquid shear flows in recessed shear coaxial injectors," Journal of Propulsion and Power, vol. 28, no. 6, pp. 1413-1424, 2012.

[14] R. J. Briggs, Electron Stream Interaction with Plasmas, MIT Press, Cambridge, Mass, USA, 1964.

[15] M. Gaster, "Growth of disturbances in both space and time," Physics of Fluids, vol. 11, no. 4, pp. 723-727, 1968.

[16] M. P. Juniper, A. Hanifi, and V. Theofilis, "Modal stability theory," in Applied Mechanics Reviews, vol. 66, 2014.

[17] F. Q. Hu, T. L. Jackson, D. G. Lasseigne, and C. E. Grosch, "Absolute-convective instabilities and their associated wave packets in a compressible reacting mixing layer," Physics of Fluids A, vol. 5, no. 4, pp. 901-915, 1992.

[18] A. G. Kulikovskii and I. S. Shikina, "Influence of viscosity on stability of tangential discontinuity in incompressible liquid," Moscow University Mechanics Bulletin, vol. 52, pp. 6-9, 1997.

[19] A. G. Kulikovskii and I. S. Shikina, "On the asymptotic behavior of localized perturbations in the presence of Kelvin-Helmholtz instability," Fluid Dynamics, vol. 20, no. 2, pp. 186-193, 1985.

[20] I. S. Shikina, "Asymptotic behavior of localized perturbations in free shear layers," Fluid Dynamics, vol. 22, no. 2, pp. 173-179, 1987.

[21] S. Pavithran and L. G. Redekopp, "The absolute-convective transition in subsonic mixing layers," Physics of Fluids A, vol. 1, no. 10, pp. 1736-1739, 1989.

[22] P. Caillol, "Absolute and convective instabilities of an inviscid compressible mixing layer: theory and applications," Physics of Fluids, vol. 21, Article ID 104101, 2009.

[23] K. Manoharan and S. Hemchandra, "Absolute/convective instability transition in a backward facing step combustor: fundamental mechanism and influence of density gradient," Journal of Engineering for Gas Turbines and Power, vol. 137, Article ID 021501, 2015.

[24] P. A. Monkewitz and P. Huerre, "Influence of the velocity ratio on the spatial instability of mixing layers," Physics of Fluids, vol. 25, no. 7, pp. 1137-1143, 1982.

[25] T. Liu, Shear-coaxial injection and mixing of cryogenic fluids under supercritical conditions [Ph.D. thesis], The Pennsylvania State University, 2007. 
[26] I. Fedioun and N. Lardjane, "Temporal linear stability analysis of three-dimensional compressible binary shear layers," AIAA Journal, vol. 43, no. 1, pp. 111-123, 2005.

[27] N. Okongo and J. Bellan, "Real-gas effects on mean flow and temporal stability of binary-species mixing layers," AIAA Journal, vol. 41, no. 12, pp. 2429-2443, 2003.

[28] M.-H. Yu and P. A. Monkewitz, "The effect of nonuniform density on the absolute instability of two-dimensional inertial jets and wakes," Physics of Fluids A, vol. 2, no. 7, pp. 1175-1181, 1990.

[29] A. Bers, "Theory of absolute and convective instabilities," in Proceedings of the International Congress on Waves and Instabilities in Plasma, Innsbruck, Austria, 1973. 


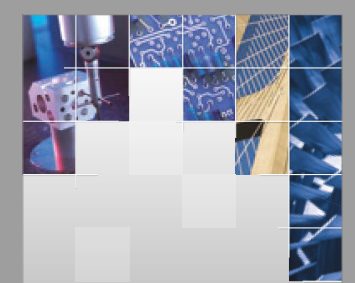

\section{Enfincering}
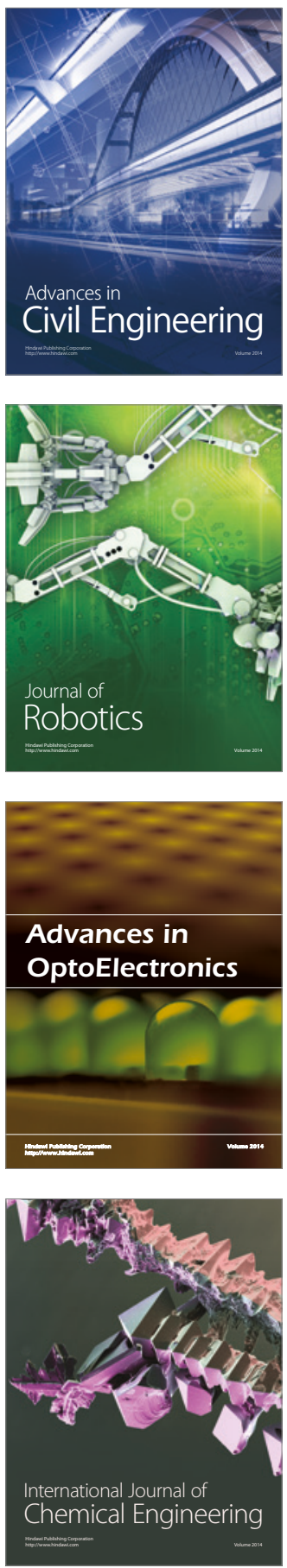

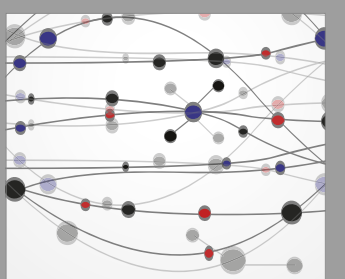

The Scientific World Journal

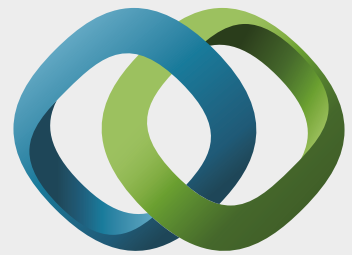

\section{Hindawi}

Submit your manuscripts at

https://www.hindawi.com
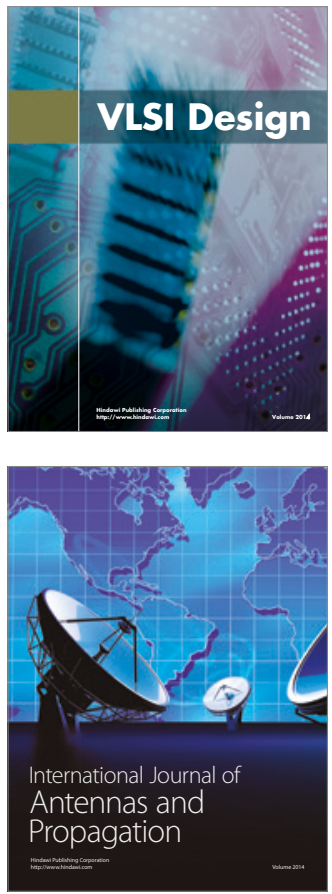

\section{Rotating}

Machinery
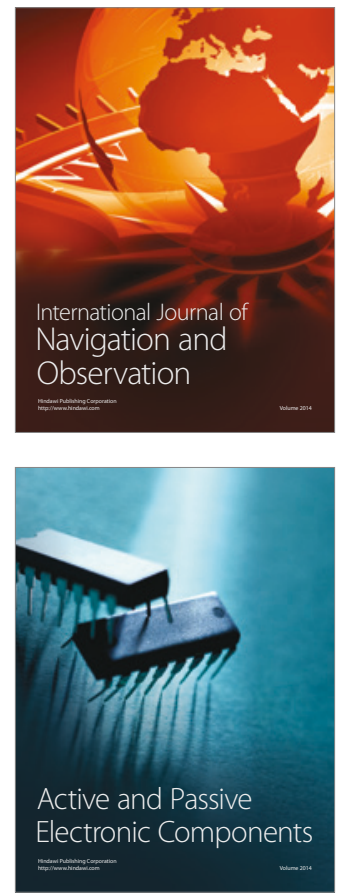
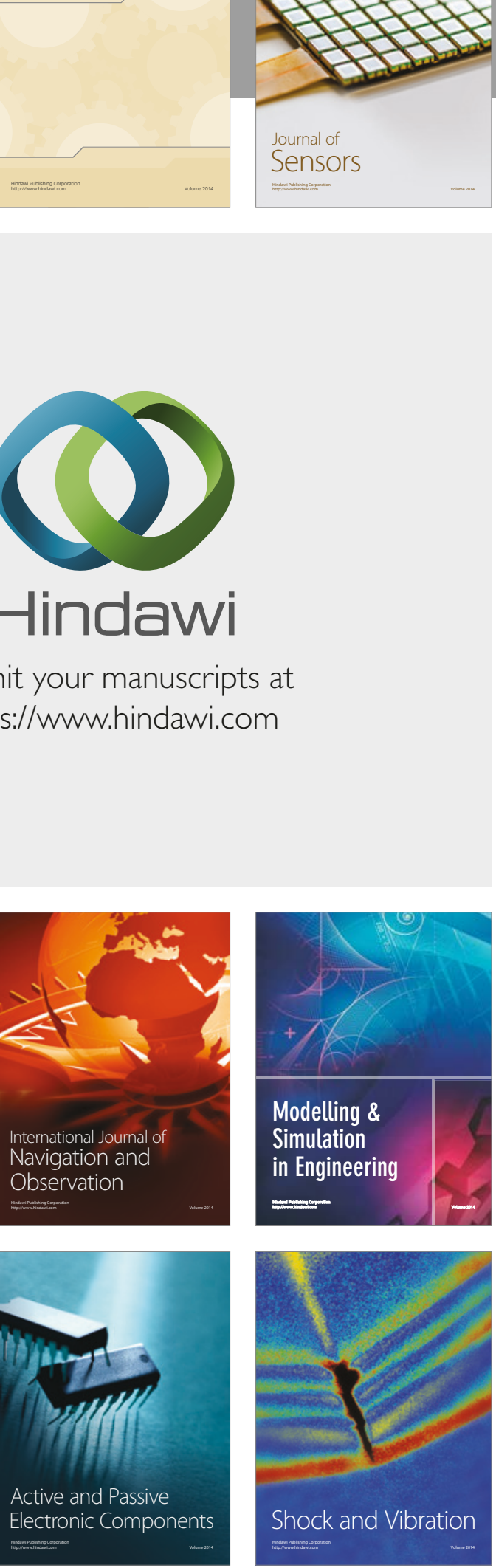
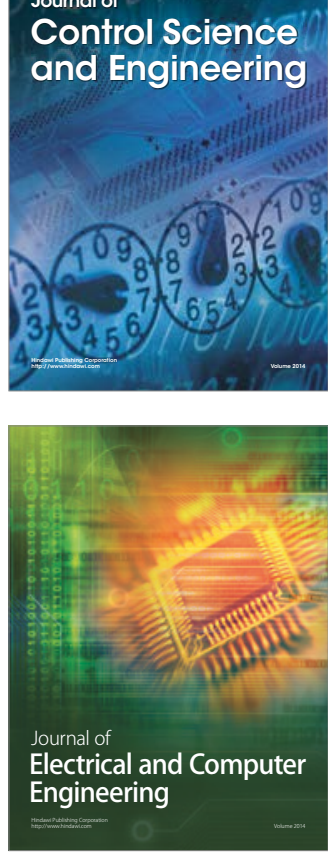

Distributed

Journal of

Control Science

and Engineering
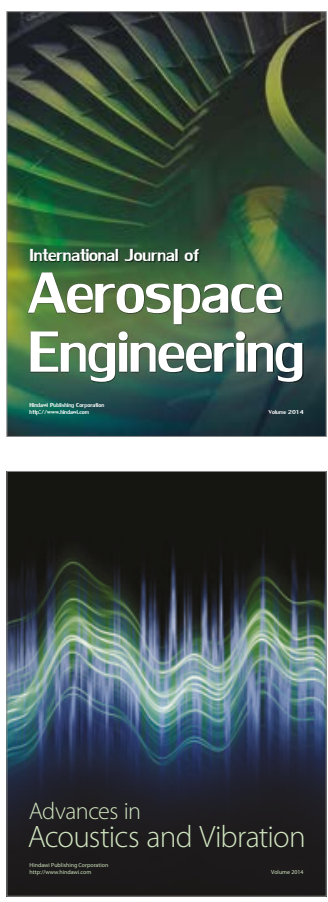

Sensor Networks 